\title{
Pre-eclampsia, eclampsia and the thrombotic microangiopathic syndromes of pregnancy
}

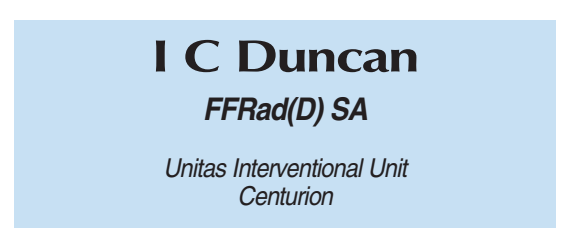

\section{Introduction}

The pregnancy-related syndromes of pre-eclampsia, eclampsia, the Haemolysis, Elevated Liver enzymes and Low Platelets or HELLP syndrome, and the Thrombotic Thrombocytopenic Purpura/Haemolytic Uraemic Syndrome (TTP/HUS) are a group of disorders sharing various clinical and pathogenetic features, principally related to endothelial damage and development of thrombotic microangiopathy triggered by pregnancy. The clinical abnormalities of these disorders include neurological disturbances, acute renal failure, hypertension, haemolysis, deranged liver enzymes, and thrombocytopenia. This review looks at the similarities and differences between these various pregnancy-related syndromes as well as some of the relevant imaging features that may sometimes be encountered by radiologists.

\section{Definitions}

Gestational hypertension is defined as blood pressure (BP) elevation of 140/90 $\mathrm{mmHg}$ or higher in a woman previously normotensive before 20 weeks' gestation, without proteinuria. This is seen in about $6 \%$ of all pregnancies. ${ }^{1,2}$

Pre-eclampsia is defined as gestational hypertension $(B P \pm 140 / 90 \mathrm{mmHg}$ ) with proteinuria $>0.3 \mathrm{~g} / 24$ hours. Pre-eclampsia is seen in about $3-8 \%$ of all pregnancies. $^{1,2}$

Severe pre-eclampsia is defined as occurring when the BP is higher than 160 -
180/110 mmHg and/or evidence of systemic involvement or end-organ dysfunction. ${ }^{1,2}$

Eclampsia is defined as the occurrence of seizures without any apparent neurological aetiology in a woman who has or who develops pre-eclampsia. About $2 \%$ of women with pre-eclampsia will develop seizures. ${ }^{1,2}$

The HELLP syndrome is defined as the presence of haemolysis, elevated liver enzymes and thrombocytopenia. Although often referred to as being severe pre-eclampsia with the additional features mentioned above, hypertension does not have to be severe or even present at all in the HELLP syndrome. HELLP syndrome occurs to some degree in about 4 - 39\% of women with pre-eclampsia. When the platelet count is $<50000 / \mu \mathrm{l}$ the syndrome is considered severe..$^{1-3}$

TTP/HUS is characterised by the presence of thrombocytopenia, haemolysis, neurological abnormalities and oliguric renal failure. Most pregnancy-related cases occur in the last trimester or postpartum period, and many have associated preeclampsia. However, only $13 \%$ of cases in women with TTP/HUS are pregnancyrelated. ${ }^{1,2}$

\section{Pathology}

The aetiology of these syndromes remains largely unclear, with several proposed theories including abnormalities of the placento-maternal unit, auto-immune disorders, endothelial cell damage and further genetic and dietary influences. ${ }^{1,4}$ The basic underlying process involves vascular endothelial cell damage. This, in turn, may be initiated by circulating factors released by the placenta secondary to ischaemic damage within the placenta itself. Substances released by the damaged endothelium such as ultra-large multimers of Von Willebrand factor (VWF) and thromboxane A2 cause platelet activation and aggregation, further activating other clotting factors and fibrin. These plateletfibrin microaggregates then lodge in the microvessels (arterioles) either blocking them completely leading to reduced tissue perfusion, or partly leading to damage of erythrocytes as they are forced through the narrowed channels. This, in turn, leads to haemolysis and is therefore termed microangiopathic haemolytic anaemia or MAHA. The endothelial cell damage also results in an abnormal pressor response to both circulating vasopressor agents (e.g. angiotensin II) and local endothelial ones (e.g. thromboxane A2, nitric oxide). This results in a generalised increase in vascular tone with resulting hypertension and vasospasm further contributing to endorgan hypoperfusion and damage. Finally the endothelial damage leads to capillary leakage with the development of tissue oedema, reduced circulating volume and haemoconcentration. These are the basic common pathomechanisms behind the pregnancy-related thrombotic microangiopathies. The various clinical syndromes are largely differentiated by the degree of severity and specificity of the end-organ damage, although the common pathomechanisms permit an explanation for the considerable degree of clinical and pathogenetic overlap between them.

\section{Clinical features}

These are largely related to the presence of vasomotor abnormalities and reduced tissue perfusion, as well as the effects of thrombocytopenia, haemolysis and anaemia, and mainly include neurological manifestations, hepatic and renal dysfunction, and coagulapathies. Although preeclampsia is diagnosed on the basis of the elevated BP and proteinuria (minimal diagnostic criteria), there are a number of other associated abnormalities that when present make the diagnosis of pre-eclampsia more certain. These include mild thrombocytopenia with a platelet count of $<100$ $000 / \mathrm{ml}$, haemolysis with an abnormal blood smear and increased lactate dehydrogenase (LDH) levels, elevated liver function 
tests (ALT, AST), epigastric pain and nausea due to liver distension, and neurological disturbances such as persistent headache or visual disturbances. Whereas mild thrombocytopenia is common in women with pre-eclampsia (15 - 29\% of cases), haemolytic anaemia is not and occurs in only about $2 \%$ of pre-eclamptic women. Haemolysis, if significant enough, is more likely to be part of HELLP or TTP/HUS. HELLP is characterised by low platelets $(<100000 / \mathrm{ml})$, haemolysis and elevated liver enzymes with associated swelling of the liver producing epigastric or right upper quadrant pain, nausea and vomiting. In rare cases spontaneous hepatic rupture may occur due to massive liver swelling. Hypertension may or may not be present with HELLP, and cerebral manifestations are rare. ${ }^{3}$ On the other hand, renal and cerebral complications are more frequently encountered in patients with TTP/HUS, ${ }^{2,5,6}$ whereas liver function disturbances are not, although these patients may still develop abdominal pain, nausea and vomiting. The neurological features associated with TTP/HUS include generalised mental status abnormalities (such as confusion, stupor or coma), focal neurological abnormalities (such as visual disturbances, aphasia, dysarthria, hemiparesis) and seizures. Hypertension is not a feature of TTP/HUS unless there is associated pre-eclampsia. ${ }^{2}$ Put simplistically, pre-eclampsia/severe pre-eclampsia may have some or all of the abovementioned clinical features plus hypertension, whereas HELLP mainly affects the liver and TTP/HUS preferentially involves the kidneys and central nervous system.

Many cases of antepartum pre-eclampsia, HELLP or TTP/HUS will resolve spontaneously. Some may persist, worsen or even change following delivery, e.g. from pre-eclampsia to HELLP or TTP/HUS. Some cases develop de novo in the postpartum period. Eclampsia developing within 48 hours after delivery occurs in approximately $25 \%$ of all eclamptics. Late postpartum eclampsia (beyond 48 hours after delivery) is very rare and can occur up to 23 days after delivery. ${ }^{7,8}$ Mortality due to eclampsia can occur in up to $13 \%$ of cases, while in the HELLP syndrome mortality ranges from $2 \%$ to $24 \% .{ }^{4}$ Between $50 \%$ and $65 \%$ of maternal deaths due to eclampsia occur as a result of cerebral haemorrhage, with other causes including hypertensive encephalopathy, cardiac arrhythmias, congestive heart failure and disseminated intravascular coagulapathy. Other complications that can occur in eclamptics include pulmonary oedema and retinal detachment. $^{4}$

Obstetric complications related to these syndromes include placental abruption and foetal distress and death.

\section{More about TTP/HUS}

TTP was first described in 1925, being characterised by fever, fluctuating neurological manifestations, renal dysfunction, thrombocytopenia and haemolytic anaemia. HUS was first described in 1955, being described primarily as a disease of childhood and also being characterised by fever, oliguric renal failure, thrombocytopenia and haemolytic anaemia. HUS occurs mainly in young children due to infection by Shiga-toxin producing enterohaemorrhagic strains of Escherichia coli. These children invariably do well clinically with supportive therapy only. ${ }^{2,5,6}$ More recently both TTP and HUS have been recognised as being different clinical presentations of a common pathological spectrum of thrombotic microangiopathy, and are now referred to jointly as TTP/HUS. There are idiopathic and familial forms of TTP/HUS, with other cases of TTP/HUS occurring secondary to cancer and chemotherapy, transplantation and immunosuppressive therapy, systemic infection, autoimmune disease, drug reactions and pregnancy. Sixty-six per cent of all patients with TTP/HUS are female. Of these cases only $13 \%$ are pregnancy related, meaning that unlike HELLP which is totally pregnancy-induced, only $8.5 \%$ of all TTP/HUS cases are pregnancy related. Some patients with TTP/HUS have been found to be severely deficient in the activity of the metalloprotease ADAMTS-13 ( $<5 \%$ normal activity)..$^{910}$ ADAMTS-13 cleaves ultra-large multimeric molecules of Von Willebrand factor (VWF) released by the endothelium. VWF is a potent activator of platelet activation and aggregation. Therefore a severe deficiency of ADAMTS13 allows uncontrolled platelet aggregation resulting in microvascular occlusion, thrombocytopenia and consumption coagulopathy. As not all patients with TTP/HUS have ADAMTS-13 deficiency there are clearly other pathogenetic factors involved that have yet to be elucidated. Pregnancy has been shown to provoke acute TTP/HUS in women with familial ADAMTS-13 deficiency, ${ }^{2,5}$ particularly in homozygotes. Without treatment TTP is fatal in $90 \%$ of patients. The only effective treatment is plasma exchange, thought to work by removing autoantibodies or other circulating pathogenetic trigger factors, as well as by replenishing absent levels of ADAMTS-13. It is therefore important to differentiate cases with TTP/HUS from those with severe pre-eclampsia and HELLP as plasma exchange is of no benefit in the treatment of the latter pathologies. ${ }^{2,5,9}$

\section{Imaging features}

These are generally related to the neurological manifestations of these pathologies. Cerebral vasospasm has been demonstrated in eclampsia and severe preeclampsia using a variety of imaging methods including transcranial Doppler (TCD), magnetic resonance angiography and conventional catheter angiography (Figs 1a, b). ${ }^{711-16}$ The true incidence of cerebral vasospasm in eclamptics and severe preeclamptics is unknown. Similar findings have also been reported in patients with HELLP, $^{3,8}$ and we have seen a single case of cerebral vasospasm associated with postpartum TTP/HUS. In addition to the abnormal pressor response of blood vessels relating to endothelial cell damage, the develop of cerebral vasospasm may also result in part due to high circulating levels of oxyhaemoglobin following breakdown of red blood cells due to the MAHA, with irritation of the vessels by the free oxyhaemoglobin itself. ${ }^{3}$ This spasm can affect intracranial vessels in an interrupted or continuous manner, and affects both large and medium-sized vessels. It mimics cerebral vasculitis angiographically..$^{12,16,17}$ As 


\section{REVIEW ARTICLE}

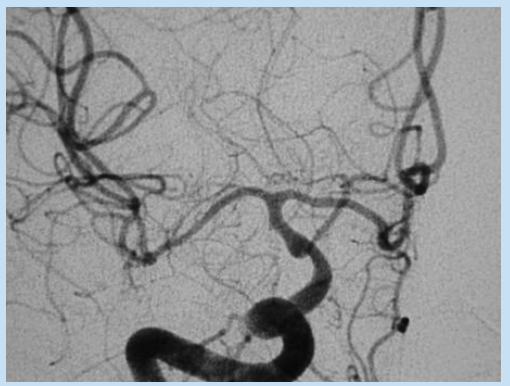

Fig. 1a. 36-year-old woman with last trimester pre-eclampsia with severe postpartum hypertension and intracerebral haemorrhage 11 days after delivery. Note the generalised spastic appearence of the central cerebral arteries at arteriography.

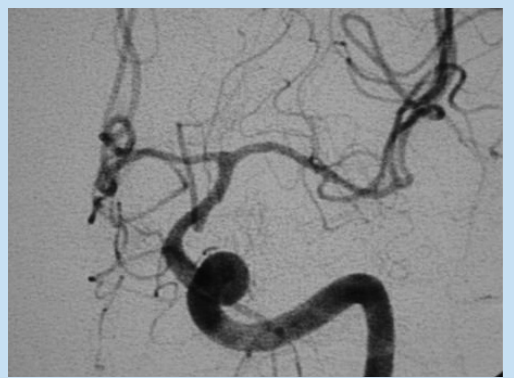

Fig. 1b. Mirror oblique projection, left internal carotid artery showing diffuse vasospasm on this side as well.

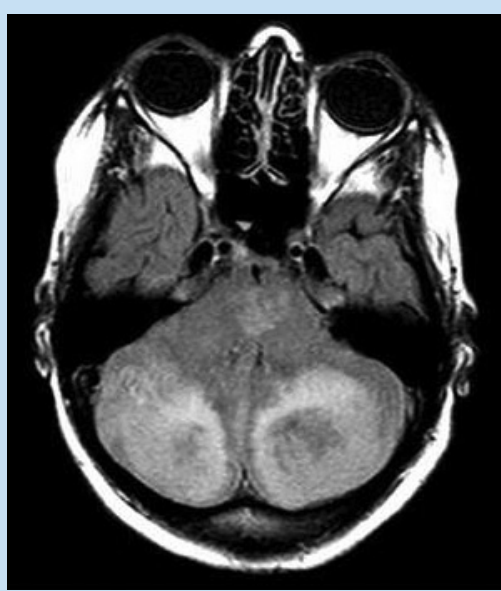

Fig. 2a. 30-year-old woman who developed HELLP syndrome and eclampsia shortly after delivery at 39 weeks. This FLAIR MR image shows areas of increased signal in the cerebellum and pons.

with the clinical symptoms the angiographic findings can spontaneously resolve within weeks of presentation. ${ }^{17,18}$ Although most cases are eventually reversible, some can develop associated cerebral infarction or haemorrhage. ${ }^{13,18}$ The other major radiolog-

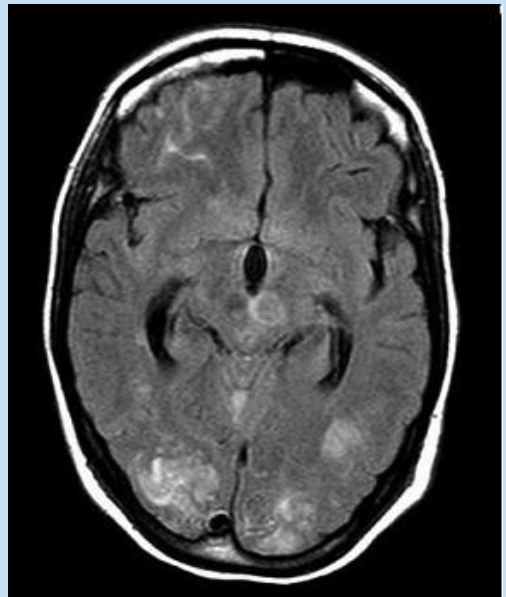

Fig. $2 b$. Similar areas of increased signal are seen in the occipital lobes and midbrain.

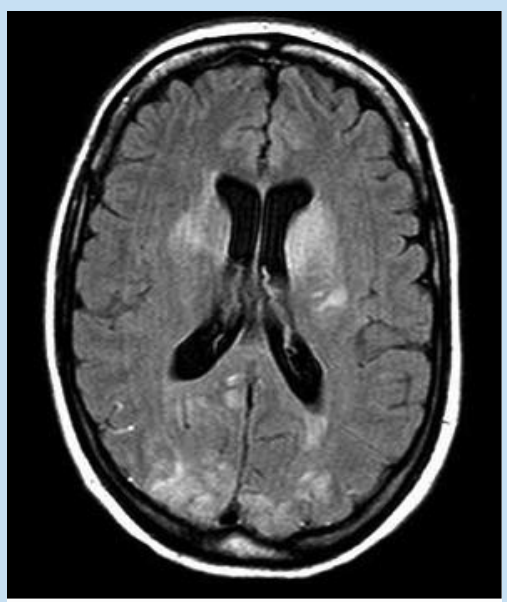

Fig. 2c. This image shows further involvement of the deep cerebral structures. There is relative sparing of the frontal and anterior parietal and temporal regions. The MR appearance is compatible with PRES, predominantly affecting the posterior brain structures.

ical abnormality seen in these cases is cerebral oedema. Hypodense areas are seen on CT scans and hyperintense areas are seen on T2-weighted and FLAIR MR images in such cases. These areas tend to be subcortical but can include the cortex, basal gangha and brainstem. Associated infarction and haemorrhage are uncommon. ${ }^{19}$ Two key differentiating features of cerebral oedema related to the pregnancy syndromes are firstly that the changes are commoner in the occipital and parietal lobes, and secondly that they are reversible. ${ }^{7,14,15,19,20}$ These features have led to coining of the term 'Posterior Reversible Encephalopathy

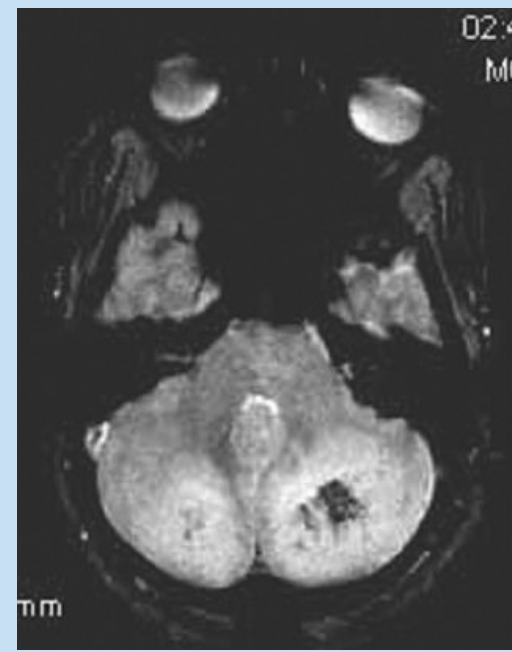

Fig. 2d. A gradient-echo sequence shows subtle early haemorrhage in both hemicerebellar hemispheres. This suggests the development of true infarctions with early haemorrhagic transformation, which is uncommonly seen in PRES. There is also severe thrombocytopenia present at this stage.

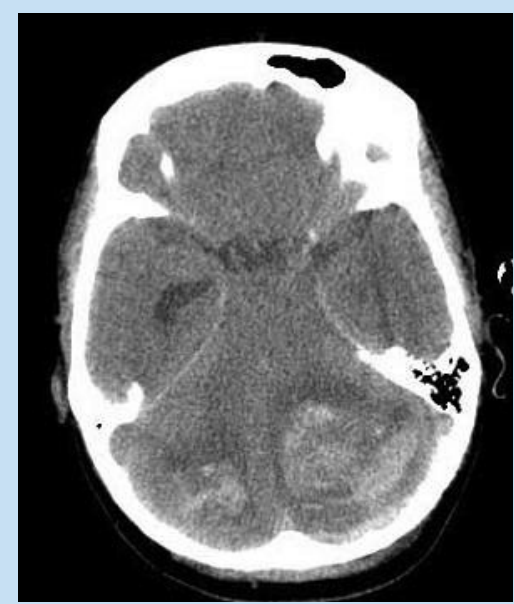

Fig. 2e. A CT scan obtained 2 days later after further neurological deterioration now shows further haemorrhagic transformation of the cerebellar lesions, leading to greater brain swelling and eventual fatal outcome.

Syndrome' (PRES). PRES is, however, not unique to pregnant patients, and has also been described in cases receiving immunosuppressive and chemotherapeutic drugs, after transplantation, in patients with hyperintensive encephalopathy, and in patients with TTP/HUS due to causes other than pregnancy. ${ }^{10,21-23}$ Despite a predilection for the posterior circulation territories, the deep structures and anterior circulation territories are also frequently involved (Figs 2a-c). The density/signal changes are said 
to be due to vasogenic oedema, showing up on T2 and FLAIR images but with negative diffusion scans. Where present diffusion abnormalities suggest progression from vasogenic to cytotoxic oedema with cell death and infarction and thus poorer clinical outcome. Subclinical haemorrhages may be seen using the appropriate gradient-echo or other blood/iron sensitive sequences (Figs 2d,e). The pathophysiology of these reversible changes is incompletely understood and is felt to be due to a failure in cerebral autoregulation, possibly secondary to endothelial damage coupled with hypertension, vasospasm and micro circulatory ischaemic damage..$^{21-23}$ The differential diagnosis for cerebral oedema and/or haemorrhage in pregnancy must include central venous/ dural sinus thrombosis and venous infarction, haemorrhage from a vascular malformation or aneurysm, arterial infarction and trauma. ${ }^{1,24}$

\section{Treatment}

The mainstay of prevention of eclampsia remains administration of magnesium sulphate. Nimodipine has been shown to be less effective in this regard..$^{25,26}$ Supportive therapy such as platelet and blood transfusions, antihypertensive agents, dialysis and early delivery may all be of benefit in severe pre-eclampsia. Highdose intravenous corticosteroid therapy is useful in HELLP syndrome. ${ }^{27}$ There is a single documented case ${ }^{28}$ of percutaneous angioplasty used to treat eclampsia-related vasospasm with good angiographic and clinical results.

\section{Conclusion}

In conclusion, the role of the radiologist in the diagnosis and management of these conditions is somewhat limited, but it is useful to have at least some understanding of their pathogenesis and in particular of the potential neurological complications and cause and recognition thereof.

\section{References}

1. Sloan MA, Stern BJ. Cerebrovascular disease in pregnancy. Current Treatment Options in Neurology 2003; 5: 391-407.

2. McMinn JR, George JN. Evaluation of women with clinically suspected thrombotic thrombocytopoenic purpura - hemolytic uremic syndrome. $J$ Clin Apheresis 2001; 16: 202-209.

3. Knopp U, Kehler U, Rickmann H, Arnold H, Gilemroth J. Cerebral haemodynamic pathologies in HELLP syndrome. Clin Neurol Neurosurg 2003, 105: 256-261.

4. Lipstein H, Lee CC, Crupi RS. A current concept of eclampsia. Am J Emerg Med 2003; 21: 223-226.

5. George JN. The association of pregnancy with thrombotic thrombocytopoeinic purpura hemolytic uremic syndrome. Curr Opin Hematol 2003; 10: 339-344.

6. Kakishita E. Pathophysiology and treatment of thrombotic thrombocytopoenic purpura/ hemolytic uremic syndrome (TTP/HUS). Int J Hematol 2000; 71: 320-327.

7. Bartynski WS, Sanghaui A. Neuroimaging of delayed eclampsia. J Comput Assist Tomogr 2003, 27: 699-713

8. Gilemroth J, Knopp U, Kehler U, Felberbaum R, Nowak G. HELLP syndrome with hemoglobin vasospasm. J Clin Neurosci 2000; 7: 58-62.

9. Vesely SK, George JN, Lemmie B, et al. ADAMTS 13 activity in thrombotic thrombocytopoenic purpura - haemolytic uraemic syndrome: relation to presenting features and clinical outcomes in a prospective cohort of 142 patients. Blood 2003; 102: 60-68.

10. Lattuada A, Rossi E, Calzarossa C, Candolfi R, Marinucci PM. Mild to moderate reduction of Von Willebrand factor cleaving protease (ADAMTS13) in pregnant women with HELLP microangiopathic syndrome. Haematologica 2003; 88: 10291034.

11. Qureshi BL, Frankel MR, Ottenlips JR, Stern BJ Cerebral vasospasm and eclampsia. Stroke 1988; 19: 326-329.

12. Trommer BL, Homer D, Mikhael MA. Cerebral vasospasm and eclampsia. Stroke 1988; 19: 326329.

13. Geraghty JJ, Hoch DB, Robert ME, Vinters HV Fatal puerperal cerebral vasospasm and stroke in a young woman. Neurology 1991; 41: 1145-1147.

14. Sengar AR, Gupta RK, Dhanuka AK, Das K. MR imaging, MR angiography and MR spectroscopy of the brain in eclampsia. Am J Neuroradiol 1997; 18:
1485-1490.

15. Lewis LK, Hinshow DB, Will AD, Hasso AN Thompson JR. CT and angiographic correlation of severe neurological disease in toxemia of pregnancy. Neuroradiology 1988; 30: 59-64.

16. Ito $\mathrm{T}$, Sakai $\mathrm{T}$, Inagawa $\mathrm{S}$, Utsu $\mathrm{M}$, Bun T. MR angiography of cerebral vasospasm in pre-eclampsia. Am J Neuroradiol 1995; 16: 1344-1346.

17. Garner BF, Burns P, Bunning RD, Laureno R. Acute blood pressure elevation can mimic arteriographic appearance of cerebral vasculitis. J Rheumato 1990; 17: 93-97.

18. Ursell MR, Marras CL, Farb R, Rowed DW, Black SE, Perry JR. Recurrent intracranial hemorrhage due to postpartum cerebral angiopathy. Stroke 1998; 29: 1995-1998.

19. Dahmus MA, Barton JR, Sibai BM. Cerebral imaging in eclampsia: Magnetic resonance imaging versus computed tomography. Am J Obstet Gynecol 1992; 157: 935-941.

20. Raroque HG, Orrison WW, Rosenberg GA Neurologic involvement in toxemia of pregnancy: Reversible MRI lesions. Neurology 1990; 40: 167169.

21. Hinchey J, Chaves C, Appignani B, et al. A reversible posterior leukoecephalopathy syndrome. N Engl J Med 1996; 334: 494-500.

22. Provenzale JM, Petrella JR, Cruz LCH, Wong JC, Engelter S, Barboriak DP. Quantitative assessmen of diffusion abnormalities in posterior reversible encephalopathy syndrome. Am J Neuroradiol 2002; 23: $1038-1048$

23. Covarrubias DJ, Luetmer PH, Campeau NG. Posterior reversible encephalopathy syndrome: Prognostic utility of quantitative diffusion-weighted MR images. Am J Neuroradiol 2002; 23: 10381048.

24. Mantello MT, Schwartz RB, Jones KM, Ahn SS, Tice HM. Imaging of neurologic complications associated with pregnancy. Am J Roentgenol 1993 150: 843-847.

25. Naidu S, Payne AJ, Moodley J, Hoffmann M Gouws E. Randomised study assessing the effect of phenyton and magnesium sulphate on maternal cerbebral circulation in eclampsia using transcranial doppler ultrasound. Br J Obstet Gynaecol 1996; 103: 111-116.

26. Belfort MA, Anthony J, Saade GR, Allen JC. A comparison of magnesium sulfate and nimodepine fo the prevention of eclampsia. N Engl J Med 2003; 348: $304-311$.

27. Martin JN jun., Thigpen BD, Rose CH, Cushman J Moore A, May WL. Maternal benefit of high-dose intravenous corticosteroid therapy for HELLP syndrome. Am J Obstet Gynecol 2003; 189: 830-834.

28. Ringer AJ, Quereshi AI, Kim S, Fessler RD, Guterman LR, Hopkins LN. Angioplasty for cerebral vasospasm from eclampsia. Surg Neurol 2001; 56: $373-379$ 